\title{
Ubiquitin sets the timer: impacts on aging and longevity
}

Article

Accepted Version

Kevei, E. and Hoppe, T. (2014) Ubiquitin sets the timer: impacts on aging and longevity. Nature structural \& molecular biology, 21 (4). pp. 290-292. ISSN 1545-9985 doi: https://doi.org/10.1038/nsmb.2806 Available at https://centaur.reading.ac.uk/65858/

It is advisable to refer to the publisher's version if you intend to cite from the work. See Guidance on citing.

Published version at: http://www.nature.com/nsmb/journal/v21/n4/full/nsmb.2806.html

To link to this article DOI: http://dx.doi.org/10.1038/nsmb.2806

Publisher: Nature Publishing Group

All outputs in CentAUR are protected by Intellectual Property Rights law, including copyright law. Copyright and IPR is retained by the creators or other copyright holders. Terms and conditions for use of this material are defined in the End User Agreement.

\section{www.reading.ac.uk/centaur}

\section{CentAUR}

Central Archive at the University of Reading

Reading's research outputs online 


\section{Left Alive with Ubiquitin: Impacts on Aging and Longevity}

Éva Kevei and Thorsten Hoppe*

Institute for Genetics and Cologne Excellence Cluster on Cellular Stress Responses in Aging-Associated Diseases (CECAD)

University of Cologne

Zülpicher Str. 47a

50674 Cologne, Germany

${ }^{*}$ Correspondence should be addressed to T.H.

*Correspondence: thorsten.hoppe@uni-koeln.de

Phone: $\quad+492214701503$

Fax: $\quad+492214703402$ 


\section{Ubiquitin sets the aging timer}

The maintenance of protein homeostasis, or proteostasis, involves the degradation of misfolded and damaged proteins and is essential for cellular function, organismal growth, and ultimately viability. The integrity of the proteome is a long-term challenge not only for individual cells but also for entire organisms since damaged and aggregated proteins accumulate with stress and aging. The ubiquitin/proteasome system (UPS) and autophagy are the major proteolytic routes embedded in a cellular quality control network that ensure efficient turnover of defective proteins. Both pathways are initiated by ubiquitylation, which provides the recognition signal for substrate selection. The ubiquitylation step is mediated by an enzymatic cascade that involves ubiquitin-activating enzymes (E1), ubiquitin-conjugating enzymes (E2), and ubiquitin protein ligases (E3), whereas deubiquitylation enzymes (DUBs) reverse this modification by removing the ubiquitin moieties ${ }^{1}$.

It is commonly thought that an age-related impairment of ubiquitin dependent proteolysis correlates with enhanced accumulation of damaged proteins, which can be cytotoxic and shorten lifespan. Beside the requirement of proteasomal integrity for normal lifespan, the identification of ubiquitin-dependent degradation pathways that specifically control the stability of lifespan regulators further represents a key role of the UPS in the aging process. Initial results provide evidence that autophagy can act as a compensatory degradation system when the UPS is impaired. However, the impact of stress and aginginduced signals on the fine-tuning of ubiquitin-dependent proteolytic pathways remains to be explored.

\section{Age-related decline in UPS function}

Cellular differentiation, developmental processes, and environmental factors challenge the integrity of the proteome in every eukaryotic cell. Accumulation of damaged and aggregated proteins is often associated with neurodegenerative disorders including Alzheimer's and Parkinson's diseases. Recent studies provide evidence that especially the progressive decline in UPS function contributes to enhanced protein aggregation and normal aging ${ }^{2}$. Initial works focused on the proteasome assembly chaperone Ump1 or the 19S subunit Rpn11 identified that both synthesis and assembly of the $26 \mathrm{~S}$ 
proteasome is required to maintain viability in yeast models of aging ${ }^{3}$. Aging is associated with the accumulation of ubiquitylated protein aggregates, which have been shown to inhibit the activity of the $26 \mathrm{~S}$ proteasome ${ }^{4}$. Thus, compromised cellular clearance of damaged proteins during aging could further aggravate the proteotoxic effect and thus reinforce the aging process. Given its functional impairment in aging, increased ubiquitindependent proteolysis might have beneficial effects on longevity. Indeed, elevated proteasomal capacity extends the replicative lifespan of budding yeast ${ }^{5}$, and the activation of the UPS extends longevity in multicellular organisms. Overexpression of the $19 S$ subunit RPN-6 or a key regulator of ubiquitin-dependent degradation, Ubiquilin, increases the lifespan and delays the onset of protein aggregate formation in both Caenorhabditis elegans and mice 6,7 . Conversely, inactivation of the ubiquitin activating enzyme (E1) Uba1 reduces the adult lifespan of Drosophila, reflecting the intricate balance between ubiquitylation and longevity ${ }^{8}$.

The spatial organization of cellular processes in eukaryotes led to the evolvement of organelle specific protein degradation pathways. For example, the turnover of misfolded proteins that accumulate in the endoplasmic reticulum (ER) involves the retrotranslocation into the cytosol for ubiquitylation, and proteasomal targeting through a process called ER-associated degradation (ERAD). Decreased expression of ERAD factors shortens the lifespan of worms ${ }^{9}$, suggesting that protein quality assurance in the ER has substantial effects on organismal homeostasis and aging. Similar to ERAD, the degradation of mitochondrial proteins is also mediated by the UPS through the mitochondria-associated degradation (MAD) pathway ${ }^{10}$. Maintenance of mitochondrial proteostasis is especially important in cellular homeostasis because damaged mitochondria produce excessive amounts of reactive oxygen species that are cytotoxic. Age-associated impairment in mitochondrial metabolism causes increased protein oxidation, which can either overload the degradation capacity of the $26 \mathrm{~S}$ proteasome or directly damage its subunits (Fig. 1). Therefore, sustained proteasomal inhibition by ROS could dramatically reduce ubiquitin-mediated turnover and further aggravate proteostasis decline ${ }^{11}$. Protein folding stress in the ER or in mitochondria induces transcriptional programs called the unfolded protein response (UPR ${ }^{E R}$ or UPR ${ }^{m t}$ ), which enhance the chaperone network and boost the degradation of misfolded proteins ${ }^{12}$. The activation of 
the UPRER, possibly involving the enhancement of ubiquitin-dependent proteolytic pathways, has recently been shown to increase the lifespan of yeast and C. elegans ${ }^{13,14}$. Consequently, the coordination of different proteolytic pathways is essential in maintaining cellular homeostasis. There is a growing interest in the use of chemical interventions that aim to preserve a youthful cellular state. The observation that rapamycin treatment delays multiple aspects of aging in different organisms by induction of autophagy has opened new paths to explore. Similarly, the identification of new drugs modulating the activity of the UPS would be extremely beneficial to develop therapeutic strategies to improve cellular proteostasis in age-related diseases.

\section{Ubiquitin-dependent regulation of longevity}

Aging is characterized by a general functional decline of cells with increased risk of disease. It is driven by stochastic accumulation of damage in biomolecules, nevertheless the functional decline is strongly influenced by genetic factors as well. The best characterized evolutionarily conserved regulatory pathways of aging are the nutrient sensing insulin/insulin-like growth factor 1 (IGF-1) signaling (IIS), the mitochondrial respiration, the caloric restriction $(\mathrm{CR})$ and the germline mediated longevity ${ }^{15}$. Interestingly, several of these longevity pathways support cellular proteostasis by transcriptional upregulation of UPS components. Recent studies provide an intriguing concept defining how IIS modulates proteasomal degradation according to spatial and temporal proteolytic demands in different organisms. Reduced insulin signaling extends lifespan from yeast to mammals, which is performed by the activation of multiple transcription factors (Fig. 1). IIS regulates the expression of the proteasome-associated DUB UBH-4/Uchl5 that normally functions as a tissue-specific inhibitor of the 26S proteasome ${ }^{16}$. This effect is mediated by the $C$. elegans FoxO transcription factor DAF16. On the other hand, the transcription factor SKN-1/Nrf2 initiates the expression of multiple proteasomal subunit genes upon proteotoxic or oxidative stress, which causes increased capacity of the $26 \mathrm{~S}$ proteasome in worms, flies, and mammals ${ }^{17}$.

Whereas a general decline of ubiquitin-dependent proteolysis contributes to aging, several studies provide evidence that direct ubiquitylation of distinct lifespan regulators also actively influences the life expectancy of different organisms. In fact, the UPS 
regulates multiple steps of IIS, affecting the stability or localization of almost all signaling components (Fig. 1). The insulin receptor, the insulin receptor substrate, the AKT kinases, the negative regulator PTEN phosphatase and the FoxO transcription factors were all shown to be targets for ubiquitylation by different E3 ligases, including CHIP, Mdm2, and Nedd $4{ }^{18}$. In mammalian cells FoxO1 ubiquitylation is regulated by Skp2-SCF and COP1. The E3 ligase MDM2 mediates ubiquitin-dependent degradation of FoxO1 and FoxO3A in response to stress. In contrast, monoubiquitylation of FoxO4 by MDM2 drives its nuclear localization and activation, which is reversed by the DUB USP7. A fine tuned balance between ubiquitylation and deubiquitylation, governed by the ubiquitin-selective chaperone CDC-48 and the deubiquitylation enzyme ATX-3 has recently been shown to regulate IIS in worms. The close interplay between both factors determines the topology of the ubiquitin chain that is optimal for proteasomal targeting of substrate proteins and modulates longevity ${ }^{19}$.

Dietary restriction (DR) is defined by the reduction in caloric intake while maintaining essential nutrient requirements. The major transcription factors important for DR-dependent lifespan extension are PHA-4/FoxA and SKN-1/Nrf2 ${ }^{15}$. The CUL-4/DDB-1 ubiquitin ligase together with WDR-23 has been shown to degrade SKN-1 and limits the lifespan of worms ${ }^{20}$. Similarly, Nrf2 is ubiquitylated by the Cul3-Keap1-E3 ligase in mammalian cells ${ }^{21}$. Another E3 ligase, WWP-1 and the E2 ubiquitin conjugating enzyme, UBC-18 have been shown to positively regulate the lifespan of DR worms in a PHA-4dependent manner. However, the direct substrate of WWP-1 is yet unknown ${ }^{22}$. DR may promote longevity by metabolic reprogramming towards increased protein degradation, thereby ameliorating the age-related impairment of both proteasomal and autophagic turnover.

The hypoxia-inducible factor 1 (HIF-1), a highly conserved transcriptional regulator of hypoxic stress response, is also involved in regulation of lifespan ${ }^{23}$. The von Hippel Lindau (VHL) E3 ubiquitin ligase negatively regulates HIF-1 by promoting its polyubiquitylation and proteasomal turnover. Although the mechanism how HIF-1 stabilization modulates the aging process is unclear, lifespan extension by the hypoxic pathway appears to be distinct from IIS and DR. 


\section{Ubiquitin's impact on aging}

Since the first discovery of its role in aging, there is growing interest in understanding the mechanistic aspects and physiological relevance of ubiquitin modifications in lifespan regulation. Recent advances in proteomic approaches opened new avenues in identifying targets of ubiquitylation; however, comprehensive studies on age-related changes of the ubiquitin-modified proteome are still missing. It is commonly accepted that the progressive impairment of the UPS contributes to natural aging. An evolving paradigm of aging research is the mutual regulation of ubiquitin-dependent proteolysis and the aging process itself but many important questions are waiting to be addressed. For example, it is unclear how detrimental the decline in proteolytic capacity is for cellular maintenance. The progressive impairment of the UPS might cause a systemic loss of cellular homeostasis, not only disintegrating cellular function but also causing death of multicellular organisms. Moreover, a genetically defined shift in the global ubiquitylation pattern of the proteome might initiate age-specific response programs. Interestingly, a recent study in worms proposed that proteostasis collapse might be a programmed event that elevates the load of damaged proteins at an early time of life, possibly representing the first incident preceding aging ${ }^{24}$. In contrast, the specific degradation of key longevity regulators provides alternative explanations for the regulation of aging and longevity governed by ubiquitin. Thereby, the overall functional decline of the UPS might even potentiate the misregulation of important lifespan pathways.

Accumulating evidence suggests that proteasomal degradation is not the only mechanism that links ubiquitylation to the aging process. A fascinating new field of research covering epigenetic regulation of longevity might involve monoubiquitylation of histone $\mathrm{H}_{2} \mathrm{~B}^{25}$. Substrate ubiquitylation can provide a variety of modification signals that render the substrate for different fates. The regulation of $\mathrm{H} 2 \mathrm{~B}$ represents only the tip of the iceberg indicating that additional unconventional roles of ubiquitin in lifespan regulation remain to be discovered. Given the mutual link between ubiquitin-dependent pathways and longevity, in-depth understanding of age-related alterations of UPS function would undoubtedly provide new opportunities for therapeutic interventions in the aging process and age-related diseases. 


\section{ACKNOWLEDGMENTS}

T.H. is supported by the Deutsche Forschungsgemeinschaft (CECAD, FOR885, SFB635, and DIP8 grant 2014376). We apologize for not having cited valuable contributions due to size limitation.

\section{COMPETING INTEREST}

The authors declare that they have no competing financial or personal interests. 


\section{REFERENCES}

1. Komander, D. \& Rape, M. Annu Rev Biochem 81, 203-29 (2012).

2. Vernace, V.A., Schmidt-Glenewinkel, T. \& Figueiredo-Pereira, M.E. Aging Cell 6, 599-606 (2007).

3. Chen, Q., Ding, Q. \& Keller, J.N. Biogerontology 6, 1-13 (2005).

4. Andersson, V., Hanzen, S., Liu, B.D., Molin, M. \& Nystrom, T. Aging-Us 5, 802812 (2013).

5. $\quad$ Kruegel, U. et al. Plos Genetics 7(2011).

6. Vilchez, D. et al. Nature 489, 263-8 (2012).

7. Safren, N. et al. PLoS One 9, e87513 (2014).

8. $\quad$ Liu, H.Y. \& Pfleger, C.M. PLoS One 8, e32835 (2013).

9. Lim, P.J. et al. Journal of Cell Biology 187, 201-217 (2009).

10. Taylor, E.B. \& Rutter, J. Biochem Soc Trans 39, 1509-13 (2011).

11. Segref, A. et al. Cell Metabolism in press (2014)

12. Digaleh, H., Kiaei, M. \& Khodagholi, F. Cell Mol Life Sci 70, 4681-94 (2013).

13. Henis-Korenblit, S. et al. Proc Natl Acad Sci U S A 107, 9730-5 (2010).

14. Heo, J.M. et al. Mol Cell 40, 465-80 (2010).

15. Kenyon, C.J. Nature 467, 622-622 (2010).

16. Matilainen, O., Arpalahti, L., Rantanen, V., Hautaniemi, S. \& Holmberg, C.I. Cell Reports 3, 1980-1995 (2013).

17. Pickering, A.M., Staab, T.A., Tower, J., Sieburth, D. \& Davies, K.J.A. Journal of Experimental Biology 216, 543-553 (2013).

18 Han, Y., Lee, H., Park, J.C. \& Yi, G.S. Molecular \& Cellular Proteomics 11(2012).

19. Kuhlbrodt, K. et al. Nat Cell Biol 13, 273-81 (2011).

20. Choe, K.P., Przybysz, A.J. \& Strange, K. Mol Cell Biol 29, 2704-15 (2009).

21. Villeneuve, N.F., Lau, A. \& Zhang, D.D. Antioxidants \& Redox Signaling 13, 1699$1712(2010)$.

22. Carrano, A.C., Liu, Z., Dillin, A. \& Hunter, T. Nature 460, 396-9 (2009).

23. Leiser, S.F. \& Kaeberlein, M. Biological Chemistry 391, 1131-1137 (2010).

24. Ben-Zvi, A., Miller, E.A. \& Morimoto, R.I. Proceedings of the National Academy of Sciences of the United States of America 106, 14914-14919 (2009).

25. Rhie, B.H., Song, Y.H., Ryu, H.Y. \& Ahn, S.H. Biochemical and Biophysical Research Communications 439, 570-575 (2013). 


\section{FIGURE LEGENDS}

Figure 1 UPS activity orchestrates the aging process. The most extensively studied genetic program regulating stress tolerance and aging is the insulin/insulin-like growth factor 1 (IGF-1) signaling (IIS) pathway. Activation of the insulin/IGF-1 receptor (IR) initiates the phosphorylation of multiple downstream kinases (PI3K, AKT), which inhibit the nuclear accumulation of the FoxO and Nrf2 transcription factors. The indicated ubiquitin-dependent degradation pathways regulate the stability of multiple signaling components of the IIS. When insulin signaling is limited, FoxO and Nrf2 translocate to the nuclei and upregulate the expression of proteasomal subunits enhancing the proteolytic capacity of the $26 \mathrm{~S}$ proteasome. In contrast, ROS production in mitochondria or aggregation of misfolded proteins in the cytosol reduces proteasomal function during aging.

IR: Insulin/IGF-1 receptor; IRS: Insulin receptor substrate; PI3K: Phosphatidyl inositol 3kinase; PIP2: phosphatidylinositol-4,5- diphosphosphate; PIP3: phosphatidylinositol3,4,5-triphosphate; PTEN: Phosphatase and Tensin homolog; AKT: AKT kinase; FoxO: Forkhead box $\mathrm{O}$ transcription factor; Nrf2: nuclear factor, erythroid 2-like 2. 


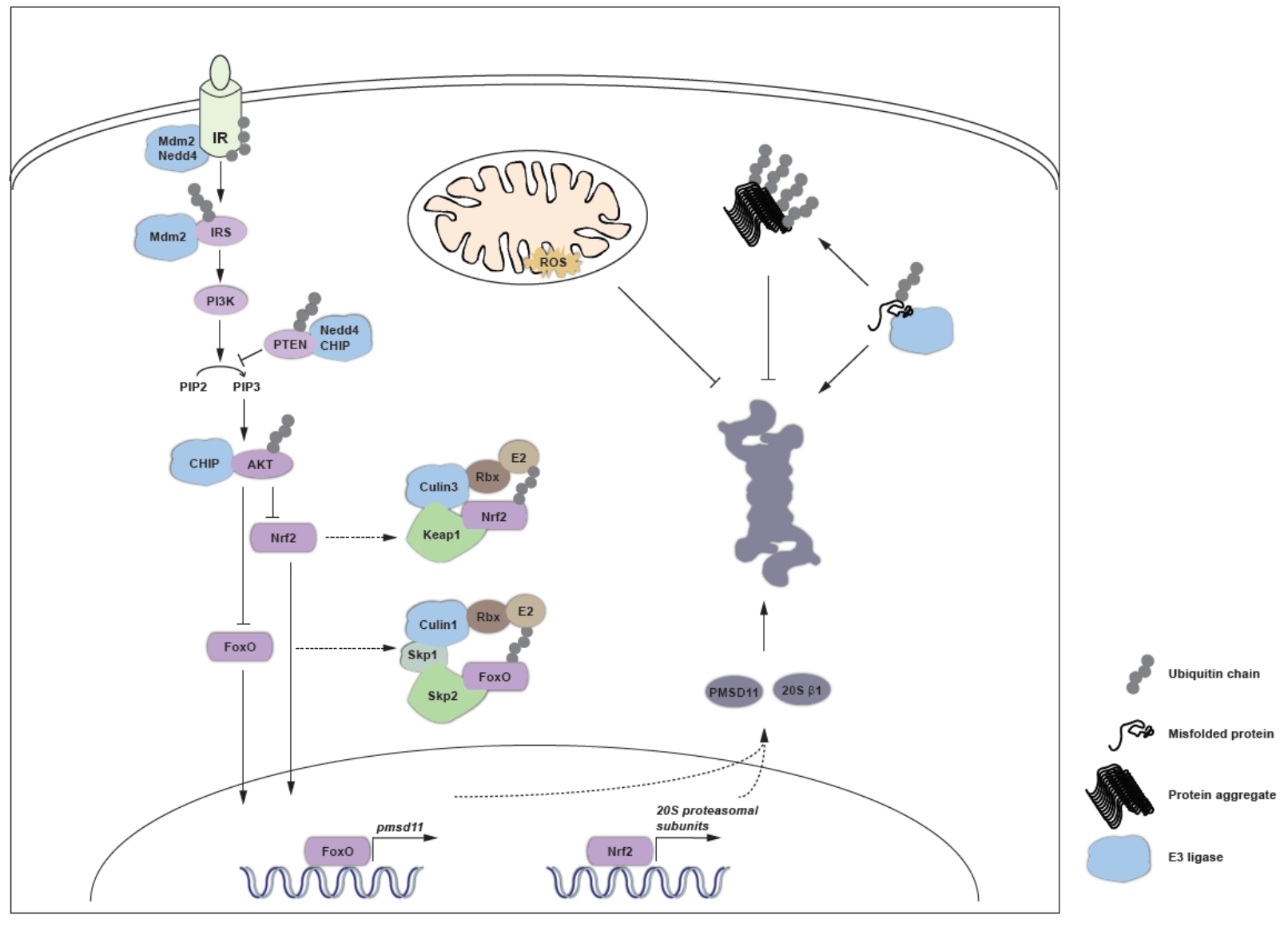

